\title{
Precise Orbit Determination of Cubesats Using Duty Cycled GPS Observations
}

\author{
Sean Lantto
}

Follow this and additional works at: https://researchrepository.wvu.edu/etd

\section{Recommended Citation}

Lantto, Sean, "Precise Orbit Determination of Cubesats Using Duty Cycled GPS Observations" (2018). Graduate Theses, Dissertations, and Problem Reports. 6035.

https://researchrepository.wvu.edu/etd/6035

This Thesis is protected by copyright and/or related rights. It has been brought to you by the The Research Repository @ WVU with permission from the rights-holder(s). You are free to use this Thesis in any way that is permitted by the copyright and related rights legislation that applies to your use. For other uses you must obtain permission from the rights-holder(s) directly, unless additional rights are indicated by a Creative Commons license in the record and/ or on the work itself. This Thesis has been accepted for inclusion in WVU Graduate Theses, Dissertations, and Problem Reports collection by an authorized administrator of The Research Repository @ WVU. For more information, please contact researchrepository@mail.wvu.edu. 


\title{
PRECISE ORBIT DETERMINATION OF CUBESATS USING DUTY CYCLED GPS OBSERVATIONS
}

\author{
Sean Lantto
}

\author{
Thesis submitted to the \\ Benjamin M. Statler College of Engineering and Mineral Resources \\ at West Virginia University \\ in partial fulfillment of the requirements for the degree of \\ Master of Science \\ in \\ Aerospace Engineering
}

Jason N. Gross, Chair Ph.D.

Thomas Evans, Ph.D.

Yu Gu, Ph.D.

Department of Mechanical and Aerospace Engineering

Morgantown, West Virginia

2018

Keywords: Orbit Determination, CubeSat, GPS, Duty Cycle, POD

Copyright (C) 2018 


\title{
ABSTRACT \\ Precise Orbit Determination of CubeSats Using Duty Cycled GPS \\ Observations
}

\author{
Sean Lantto
}

GPS can provide and has provided down to sub-centimeter accurate precise orbit determination (POD) for low Earth orbit (LEO) spacecraft. This is achieved using carrier-phase measurements, and for maximum accuracy, continuous tracking is needed to resolve the unknown constant carrier cycle ambiguities. Continuous GPS tracking can be difficult to obtain on small satellites, such as CubeSats, due to their stringent onboard power resources. This often leads to the GPS receiver power duty cycling during operations to allow for more on-board power to be used for other scientific instruments. This thesis investigates the sensitivity of GPS solution accuracy to duty cycled observations. In addition, it considers combining the duty cycled GPS observables with a simple dynamic model through an extended Kalman Filter (EKF) to maintain high accuracy POD despite duty cycling. Tests are conducted on simulated observables which are generated for 10 different satellite orbit configurations and varying amounts of GPS receiver duty cycle. Comparisons between, a simple GPS kinematic tracking and a two-body reduced dynamic approach are investigated, and the twobody reduced dynamic approach is shown to yield a 3D root mean square error (RMSE) that is significantly better than the kinematic approach, primarily during the initial solution convergence period. This thesis also investigates the effect of modeling the Earth's oblateness when integrating the state of the spacecraft in concert with a two-body model to propagate the error-covariance of the state. 


\section{Acknowledgments}

I would like to thank my advisor Dr. Jason Gross, for all of his assistance on this work, and for helping me get involved with STF-1 during my time as an undergraduate, and motivating me to pursue my Masters, without which I likely would not have had the many opportunities I do now.

I would like to thank the NASA West Virginia Space Grant Consortium (WVSGC) for providing me with a Graduate Research Fellowship allowing me to undertake this work. I would also like to thank NASA IV\&V in Fairmont, WV for providing the opportunity to work with STF-1's hardware and with providing access to a GPS simulator to advance this research. 


\section{Contents}

$\begin{array}{ll}\text { Abstract } & \text { ii }\end{array}$

Acknowledgments $\quad$ iii

List of Figures $\quad$ vi

List of Tables $\quad$ vii

1 Introduction 1

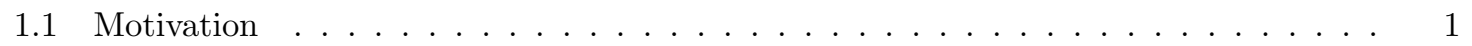

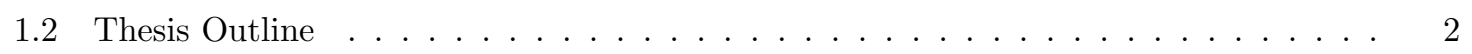

2 Literature Review $\quad 4$

2.1 Precise Orbit Determination . . . . . . . . . . . . . . . . . . . . 4

2.1.1 Reduced Dynamic POD . . . . . . . . . . . . . . . . . 5

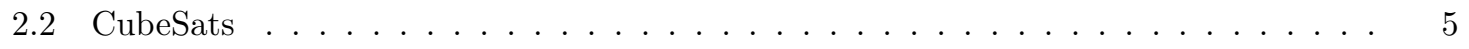

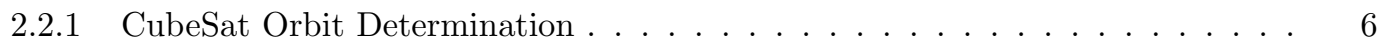

3 Background Information $\quad 8$

3.1 Astrodynamics and Force Models . . . . . . . . . . . . . . . . . . 8

3.1 .1 Two-Body Problem $\ldots \ldots \ldots \ldots \ldots \ldots$

3.1.2 Perturbed Orbits . . . . . . . . . . . . . . . . . . . . . . . 12

3.2 Global Positioning System . . . . . . . . . . . . . . . . . . . . . . 14

3.3 Kalman Filters . . . . . . . . . . . . . . . . . . . . . . . . . . . 15

$3.3 .1 \quad$ EKF Overview . . . . . . . . . . . . . . . . 15

3.3.2 Equations for Reduced Dynamic POD EKF . . . . . . . . . . . . . . 16 
4 Technical Approach $\quad 18$

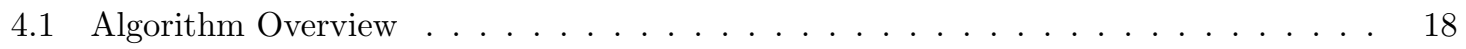

4.2 State Transition Matrix Formulation . . . . . . . . . . . . . . . . . . . . . . . . . 19

4.2.1 Two-Body State Transition Matrix . . . . . . . . . . . . . . . . . . . 19

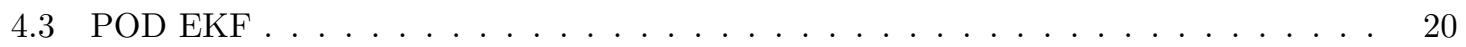

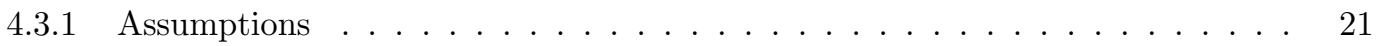

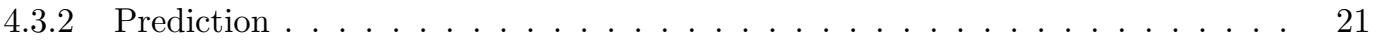

4.3.3 Measurement Update ........................ 22

5 Simulation Setup $r$

5.1 Orbit Selection . . . . . . . . . . . . . . . . . . . . . . 23

5.2 Low Earth Orbit Simulation . . . . . . . . . . . . . . . . . . . . . . . 24

5.3 GPS Observable Simulation . . . . . . . . . . . . . . . . . . . . . . 24

5.4 Hardware in the Loop Testing . . . . . . . . . . . . . . . . . . . . . . . . . . . 25

5.5 Sensitivity Study Design . . . . . . . . . . . . . . . . . . . . . 26

$\begin{array}{llr}6 & \text { Results } & 27\end{array}$

6.1 Kinematic . . . . . . . . . . . . . . . . . . . . . . . . . 28

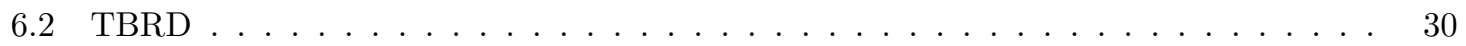

6.3 Comparison ................................. 32

6.4 STF-1 Orbit with Drag and Solar Radiation Pressure Perturbations . . . . . . . . . 32

7 Conclusions \& Future Work

7.1 Conclusions ............................... 35

7.2 Future Work .............................. 35 


\section{List of Figures}

1.1 STF-1 prior to thermal vacuum and vibration testing. (Image courtesy of Matt Grubb

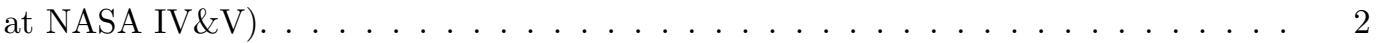

5.1 Number of satellites over one on cycle using the Spirent GPS simulator. . . . . . . . 26

5.2 Number of satellites over one on cycle in Matlab simulation. . . . . . . . . . . . . 26

6.1 RMS Errors for various duty cycle times for pure kinematic approach $\ldots \ldots \ldots$

6.2 Mean RMS Errors for various duty cycle times for pure kinematic approach . . . . . 29

6.3 RMS Errors for various duty cycle times for TBRD approach . . . . . . . . . . . . 30

6.4 Mean RMS Errors for various duty cycle times for the TBRD approach after 30 seconds of convergence. . . . . . . . . . . . . . . . . . . . 31

6.5 Mean RMS Errors for various duty cycle times for the TBRD approach. . . . . . . . 31

6.6 Difference of median RMS errors for various duty cycle times for the TBRD approach after 30 seconds of convergence. . . . . . . . . . . . . . . . . . . 32

6.7 RMS Errors for various duty cycle times for kinematic, TBRD, and full gravity ap-

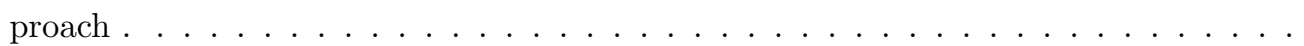

6.8 3D position integration error for all investigated POD approaches during GPS off-

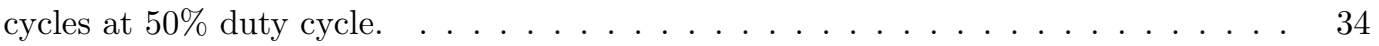

6.9 3D position Error for all except the kinematic POD approaches during GPS on-cycles at $50 \%$ duty cycle. . . . . . . . . . . . . . . . . . . . . 


\section{List of Tables}

5.1 Investigated Satellite Orbital Parameters . . . . . . . . . . . . . . . . . . . . 24

5.2 Sensitivity Study, EKF Input Parameters _ . . . . . . . . . . . . . . . . 26

6.1 Root Mean Square Error across all 10 Satellites for $10 \%$ increments of a 10 Minute Duty Cycle, when GPS is on, Kinematic estimator . . . . . . . . . . . . . . 29

6.2 Root Mean Square Error across all 10 Satellites for $10 \%$ increments of a 10 Minute Duty Cycle, when GPS is on, TBRD estimator . . . . . . . . . . . . . 30

6.3 Difference between kinematic and TBRD median RMSE after 30 seconds of convergence. 32

6.4 Root Mean Square Error for POD when using STF-1 orbit with drag and solar radiation pressure perturbations when GPS is on. . . . . . . . . . . . . 34 


\section{Acronyms}

$\begin{array}{ll}\text { GNSS } & \text { Global Navigation Satellite System } \\ \text { GPS } & \text { Global Positioning System } \\ \text { LEO } & \text { Low Earth Orbit } \\ \text { NASA } & \text { National Aeronautic and Space Administration } \\ \text { POD } & \text { Precise Orbit Determination } \\ \text { STF-1 } & \text { Simulation to Flight } 1 \\ \text { TBRD } & \text { Two-Body Reduced Dynamic } \\ \text { WVU } & \text { West Virginia University }\end{array}$




\section{Chapter 1}

\section{Introduction}

Part of this thesis is reproduced from my conference paper [1].

\subsection{Motivation}

Orbit determination is the estimation of a spacecraft's, or other orbiting body's, orbital parameters from some set of observations $[2,3]$. Precise orbit determination seeks to describe an orbit quantitatively with precision and accuracy [4], where these are dictated by mission requirements and with the current tech capable of reaching millimeter level. GPS has been used for precise orbit determination (POD) of spacecraft since first demonstrated on TOPEX/Poseidon [5]. Centimeter level accurate POD is achievable using the carrier phase measurements of the global positioning system (GPS) signal. Use of the carrier phase measurements requires solving for an ambiguity term [6], which requires the continuous tracking of GPS satellites.

Due to stringent power requirements (i.e. limited volume for batteries and limited surface area for solar panels), CubeSats often can not support continuous GPS receiver operation, requiring the receiver to be power duty cycled. Duty cycling allows the power requirements to be met at the consequence of having to resolve the carrier-phase ambiguities each time the receiver is turned on.

Simulation to Flight 1 (STF-1) [7] is a $3 \mathrm{U}$ CubeSat built at NASA Independent Verification and Validation (IV\&V) facility in Fairmont, WV to demonstrate the capability of using software only simulations during the developmental life-cycle of a CubeSat. STF-1 hosts multiple payloads experiments provided by West Virginia University (WVU), one of which is a NovAtel OEM615 dual-frequency GNSS receiver. To conserve on-board power, the STF-1 GPS receiver will be power duty cycled during nominal operations. Duty-cycling of the GPS receiver is expected to reduce 


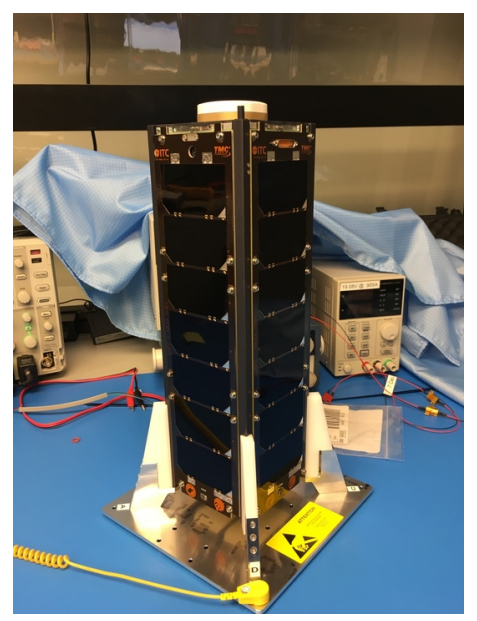

Figure 1.1: STF-1 prior to thermal vacuum and vibration testing. (Image courtesy of Matt Grubb at NASA IV\&V).

the overall accuracy of the precise orbit determination, due to the fact that, upon each power cycle, the carrier-phase ambiguities become unknown and must be resolved again. Resolving new ambiguities over short observational durations is not favorable [6]. This thesis demonstrates a method of achieving decimeter level POD, using the duty cycled GPS measurements. To alleviate the negative consequences of the duty cycling on the GPS measurements, a dynamic model of the space craft is used to provide an estimate of the spacecraft's position and velocity. This model is updated with the GPS measurements when the receiver is on. This combination of a dynamic model with kinematic measurements is known as a reduced dynamic approach $[8,5]$, and is discussed in detail in the following chapter. Due to the limited computational resources of a CubeSat, a simplified version of the reduced dynamic approach to POD is taken, using a simple two-body model to estimate the spacecraft's state. This approach is tested on simulated data (orbits, GPS observables, etc), though will be tested with flight data when STF-1 launches and returns data.

\subsection{Thesis Outline}

The second chapter of this thesis provides a review of the literature pertaining to orbit determination and the global positioning system's role in it. It will also provide needed background on the orbit determination process, the global positioning system, as well as Kalman filtering. The third chapter describes the technical approach taken, explaining how the reduced dynamic approach was applied. Chapter 4 discusses how the simulated data was created to test the reduced dynamic approach. The results from testing on the simulated data are discussed in chapter 5 , where the results from the 
reduced dynamic approach are compared to a purely kinematic estimator. Finally chapter 6 presents the conclusions drawn, and discusses potential future work to expand on this thesis. 


\section{Chapter 2}

\section{Literature Review}

\subsection{Precise Orbit Determination}

This section provides a review of the orbit determination process, including a review of astrodynamics and the forces acting on a spacecraft in orbit, GPS observation models, and specific cases of POD for CubeSats.

Orbit determination is the estimation of a satellite's state at some time from a series of observations $[3,9]$. There are several approaches to orbit determination, this work focuses on POD using GPS observations as opposed to other observation sources such as radar, radiometric, and laser tracking. GPS based POD can be broken into several approaches as well, including a kinematic approach, a dynamic approach, and a reduced dynamic approach [10, 11].

A kinematic approach to POD requires no a priori information of a spacecraft's state, and no description of it dynamics. This approach is very sensitive to poor view geometry, bad measurements, and outages/data gaps [8], which are inevitable when power duty cycling. An example of kinematic POD is given by [4], where a sequential least-squares filter/smoother was developed that only uses data from the GPS receiver on the spacecraft and the IGS GPS constellation ephemeris and clock data products, no dynamic model.

Dynamic POD use known precise models of the forces acting on a spacecraft, limited by modeling errors such as atmospheric drag model errors. The reduced dynamic approach, first proposed in [12], uses a process noise model representing a fictitious force (aka. empirical accelerations[8]) acting on the spacecraft to handle dynamic modeling errors. 


\subsubsection{Reduced Dynamic POD}

The reduced dynamic approach to POD was first proposed in [12], and first demonstrated on the TOPEX/Poseidon mission [5]. This approach uses GPS measurements to make geometric corrections for errors due to mis-modeling of a dynamic model, at the cost of increased measurement error. Both kinematic and dynamic state transition methods are used with some relative weight, controlled by adjusting three process-noise parameters representing a fictitious force, though [12] states that one could also add process noise to the spacecraft's state itself.

Yunck et.al.[12,5] present a Kalman filter based approach to the reduced dynamic approach, where the state vector becomes $\mathbf{X}=[\mathbf{x}, \mathbf{p}]$. Here $\mathbf{p}$ is the three dimensional fictitious force. These equations can be found in the Kalman filter section of the background chapter.

In [5], a 29 day interval was investigated, and the reduced dynamic solutions were assessed using postfit phase residuals, formal errors, a comparison with GPS dynamic solutions, comparison with laser and Doppler dynamic solutions, and altimetry closure and crossover agreement. It was found that the formal errors for reduced dynamic solutions were below $2 \mathrm{~cm}$ and altitude error was estimated at $3 \mathrm{~cm}$ RMS. Both dynamic and reduced dynamic solutions were accurate to better than $5 \mathrm{~cm}$ RMS, but the paper states that with a spacecraft at lower altitudes than TOPEX, the reduced dynamic should degrade very little.

A comparison between applying the reduced dynamic approach to both a batch least squares estimator and an extended Kalman filter/smoother is made in [8]. As opposed to using he firstorder Gauss-Markov process used in the $\operatorname{EKF}[5,12,8]$, the batch least squares estimator piece-wise empirical accelerations are adjusted in consecutive sub-intervals. This comparison was done using GPS measurements from the GRACE mission. Using dual-frequency ionosphere-free pseudorange and carrier phase measurements reconstructed the GRACE trajectory down to $4 \mathrm{~cm}$ 3D RMS, and when only using singl-frequency down to $7 \mathrm{~cm}$ 3D RMS. Similar accuracy was achieved with both the batch least-squares and EKF. The EKF proved to use memory and processing time more efficiently, while the least-squares exhibited better robustness in case of data gaps, and provides better smoothness of the trajectory.

\subsection{CubeSats}

CubeSats came about as a part of a collaboration between professors at California Polytechnic State University and Standford University's Space Systems Development Lab to provide a standard 
design for picosatellites $[13,14]$, providing a low cost platform for universities and organizations to do space experiments. A CubeSat is said to be a $10 \mathrm{~cm}$ cube with a mass of about 1.33 kilograms, though CubSats may be stacked into multiple configurations such as a $3 \mathrm{U}$ or 3 unit CubeSat or a $6 \mathrm{U}$ (6 unit) CubeSat. CubeSats are launched as secondary payloads on launch vehicles, though some launch vehicles are being designed with only small satellites in mind $[15,16]$. An alternative for CubeSat deployment is to send the CubeSat to the International Space Station, where it may be deployed from the Japanese Experiment Module (JEM), using the JEM Small Satellite Orbital Deployer (J-SSOD) [17, 18].

\subsubsection{CubeSat Orbit Determination}

A method of scalable orbit determination for fleets of small satellites was presented by Planet Labs Inc. using two-way UHF radio ranging [19]. The two-way time of flight range measurements are fit to an orbit using a numerical force model and a high precision propagator, which then propagates into the future to produce a two-line element set (TLE). They compare their in-house OD method with the Joint Space Operations Center (JSpOC) TLEs. Both the JSpOC and ranging OD solutions are compared to a "truth" ephemeris derived from GPS measurements taken on satellites carrying experimental software defined GPS receivers. For post-processed solutions the position offset of the ranging and JSpOC TLEs from the GPS ephemeris had an RMS if $0.45 \mathrm{~km}$ and $1.45 \mathrm{~km}$ respectively with maximums of $0.86 \mathrm{~km}$ and $3.46 \mathrm{~km}$. When used to predict future ephemeris the ranging method had an RMS of $0.56 \mathrm{~km}$ with a maximum of $1.03 \mathrm{~km}$, while the JSpOC TLEs had an RMS of $1.82 \mathrm{~km}$ and a maximum of $3.84 \mathrm{~km}$ position off set when compared to post processed GPS derived ephemeris.

Project LEDsat, an international project, is designed to help improve orbit determination and identification fo CubeSats in LEO. Multiple CubeSats containing multiple methods of position measurement; including GPS, optical tracking, satellite laser ranging (SLR), and radio tracking; making it possible to compare the different methods of orbit determination. All of the CubeSats will contain LEDs for optical tracking, and if each LEDsat has different LED flash patterns, identification of closely spaced satellites will be possible. So far the Sapienza University of Rome has launched a $3 \mathrm{U}$ CubeSat with LED's and retro-reflectors, University of Michigan is designing a 3U CubeSat with LEDs, GPS, SLR, and radio tracking (ODsat), and the Royal Military College of Canada is producing a Canadian LEDsat[20]. Sapienza will be launching a follow on $1 \mathrm{U}$ CubeSat containing both LEDs and laser retro-reflectors. They cite that the Japanese CubeSat FITSAT-1 demonstrates that LEO spacecraft can be tracked with small ground-based telescopes, and state that the LEDs 
on these satellites can be used for telemetry and optical communication as well.

The Braunshweig Educational Orbital CubeSat, or BeoCube, is the first CubeSat from TU Braunschweig, and contains a payload known as Braunschweig Experimental Payload, or BePod. BePod is a software defined Global Navigation Satellite System (GNSS) receiver, capable of supporting multiple GNSS signals, such as GPS, the European Galileo navigation system, or other systems on the L1 frequency. A secondary payload of a scaled down laser retro-reflector, known as the Laser Ranging Experiment (LaREx), will verify the GNSS accuracy[21].

The CanX-2 mission was the first to successfully demonstrate dual-frequency navigation on board a CubeSat[22] with on-board navigation solutions exhibiting position errors on the $10 \mathrm{~m}$ to $100 \mathrm{~m}$ level, and velocity errors of $0.1 \mathrm{~m} / \mathrm{s}$ to $0.5 \mathrm{~m} / \mathrm{s}$. Using data from the CanX-2 telemetry, short arc orbit solutions were estimated to an accuracy of $1 \mathrm{~m}$, and the long arc solutions, that included bridging GPS outages were accurate within $10 \mathrm{~m}$ to $100 \mathrm{~m}$, though the receiver had to be active for at least half an orbit per day.

The Radio Aurora eXplorer (RAX) CubeSat mission launched 2 CubeSats containing a GPS subsystem to provide time within 1 microsecond and position within 1 kilometer [23]. The position accuracy (standard deviation errors), were determined from the BESTXYZ log from the NovAtel OEMV-1-L1 receiver used on the spacecraft operating at $0.2 \mathrm{~Hz}$, was found to have a mean of 2.89 $\mathrm{m}$ and a with a maximum of $4.02 \mathrm{~m}$, well within the mission requirements.

Work done by Yang et. al. [24] proposes a GPS and BeiDou Navigation Satellite System (Beidou) precise point positioning (PPP) orbit determination technique, and tests it on a ground testbed using GNSS data from the International GNSS monitoring and Assessment Service (iGMAS) and MultiGNSS Experiment (MGEX). The data is processed using static and kinematic PPP and it was shown that decimeter accuracy was achievable. These ground tests will support a future LEO CubeSat mission proposed jointly by Beijing Aerospace Control Centre and China Spacesat Co. Ltd. 


\section{Chapter 3}

\section{Background Information}

\subsection{Astrodynamics and Force Models}

An understanding of astrodynamics is necessary for orbit determination. The following sections review basic astrodynamics (two-body problem), as well as the perturbing forces acting on a spacecraft, and how they impact its orbit.

\subsubsection{Two-Body Problem}

The two-body problem, also known as Kepler's problem, is the study of satellite motion, and was first solved by Issac Newton, validating Kepler's laws. The acceleration of a spacecraft can be given by Newton's law of gravity, with the assumption that the Earth is spherically symmetric and that the spacecraft's mass is negligible compared to Earth's mass [2].

$$
\ddot{\mathbf{r}}=-\frac{G M}{r^{3}} \mathbf{r}=-\frac{\mu \mathbf{r}}{r^{3}}
$$

In Eq. 3.1, $\mathbf{r}$ is the spacecraft's position vector, $r$ is the normalized distance from the center of mass of the planet being orbited. $\mu$ is the gravitational parameter, which is the product of the gravitational constant $(G)$ and the planets mass $(M)$. From Eq. 3.1, the elements describing a spacecraft's orbit can be found, the derivation of which can be found in multiple texts including $[2,25]$.

Five elements can be used to describe an orbit. Those are semi-major axis (a), eccentricity 
$(e)$, inclination $(i)$, right ascension of the ascending node $(\Omega)$, and the argument of periapsis $(\omega)$. The spacecraft's position along the orbit is defined by the mean anomaly $(M)$, which is the mean motion(Eq. 3.2) times some $\Delta t$.Thus a total of six elements are required to describe the motion of a spacecraft. However to obtain the position and velocity of the spacecraft in an inertial coordinate frame from these six elements requires solving Kepler's Equation 3.3 to obtain the eccentric anomaly $(E)$.

$$
\begin{gathered}
n=\sqrt{\frac{\mu}{a^{3}}} \\
E(t)-e \sin (E(t))=n\left(t-t_{p}\right)=M
\end{gathered}
$$

In Eq. $3.3, t_{p}$ is the time of periapsis passage. Solving Kepler's Equation for $E$ can only be done by iterative methods and Newton's method is commonly used to do so, though other methods do exist [2]. To employ Newton's method, an initial value $E_{0}=M$ is selected, and an the auxiliary function Eq. 3.4 is defined. The solution is equivalent to finding the root of $f(E)$ for some $M$.

$$
f(E)=E-e \sin (E)-M
$$

Newton's method (Eq. 3.5) is iterated until $E_{i}$ changes by less than some pre-determined amount.

$$
E_{i+1}=E_{i}-\frac{f\left(E_{i}\right)}{f^{\prime}\left(E_{i}\right)}=E_{i}-\frac{E_{i}-e \sin \left(E_{i}\right)-M}{1-e \cos \left(E_{i}\right)}
$$

Once the eccentric anomaly is calculated, the position and velocity can be found in the perifocal coordinate system (aligned with rotation axis and equator), using Eqs. 3.6 through 3.9.

$$
r_{P Q W}=[a \cos (E)-e, a \sqrt{(1-e)(1+e)}, 0]
$$




$$
R=a(s-e \cos (E))
$$

$$
V=\frac{\operatorname{sqrt}(\mu * a)}{R}
$$

$$
v_{P Q W}=[-V \sin (E), V * \sqrt{(1-e)(1+e)}, 0]
$$

The position and velocity in the perifocal coordinate frame can be rotated into the inertial frame using the rotation matrix $P Q W$, whose columns are the Gaussian vectors, shown in Eq. 3.10 and multiplying against the perifocal position and velocity vectors as shown in Eq. 3.11.

$$
\begin{aligned}
& P Q W=\left[\begin{array}{ccc}
\cos (\omega) \cos (\Omega)-\sin (\omega) \cos (i) \sin (\Omega) & -\sin (\omega) \cos (\Omega)-\cos (\omega) \cos (i) \sin (\Omega) & \sin (i) \sin (\Omega) \\
\cos (\omega) \sin (\Omega)+\sin (\omega) \cos (i) \cos (\Omega) & -\sin (\omega) \sin (\Omega)+\cos (\omega) \cos (i) \cos (\Omega) & -\sin (i) \cos (\Omega) \\
\sin (\omega) \sin (i) & \cos (\omega) \sin (i) & \cos (i)
\end{array}\right] \\
& r_{E C I}=P Q W * r_{P Q W} \\
& v_{E C I}=P Q W * v_{P Q W}
\end{aligned}
$$

Just as a unique inertial position and velocity can be found from the orbital elements, a unique set of elements themselves can be found from the position and velocity. This allows the propagation of the spacecraft's position and velocity in time by solving Kepler's equation. To find the orbital elements of the spacecraft from a position and velocity state, one must first determine the specific angular momentum (aka the areal velocity) using Eq. 3.12, which can then be used to find $i$ and $\Omega$ using Eqs. 3.13 [2].

$$
\mathbf{h}=\mathbf{r} \times \dot{\mathbf{r}}=\left(\begin{array}{c}
y \dot{z}-z \dot{y} \\
z \dot{x}-x \dot{z} \\
x \dot{y}-y \dot{x}
\end{array}\right)
$$




$$
i=\arctan \left(\frac{\sqrt{\left(\frac{h_{x}}{h}\right)^{2}+\left(\frac{h_{y}}{h}\right)^{2}}}{\left(\frac{h_{z}}{h}\right)}\right), \Omega=\arctan \left(\frac{\left(\frac{h_{x}}{h}\right)}{-\left(\frac{h_{y}}{h}\right)}\right)
$$

The areal velocity $h$ can then be used to find the semi-latus rectum, $p$ (Eq. 3.14), and the vis-viva law can be used to find the $a$ (Eq. 3.15) [2].

$$
\begin{gathered}
p=\frac{h^{2}}{\mu} \\
a=\left(\frac{2}{r}-\frac{v^{2}}{\mu}\right)^{-1}
\end{gathered}
$$

The semi-major axis and the semi-latus rectum may be used to find the eccentricity $e($ Eq. 3.16) and the mean motion $n .($ Eq. 3.2) [2].

$$
e=\sqrt{1-\frac{p}{a}}
$$

The eccentric anomaly $E$ may now be solved for using Eq. 3.17, which can be used in Kepler's equation(Eq. 3.3) to find the mean anomaly $M[2]$.

$$
E=\arctan \left(\frac{\left(\frac{\mathbf{r} \cdot \dot{\mathbf{r}}}{a^{2} n}\right)}{1-\frac{r}{a}}\right)
$$

The final element, the argument of periapsis $\omega$, requires that the argument of latitude $u$ is first determined using Eq. 3.18, as well as the true anomaly of the spacecraft is determined using Eq. $3.19[2]$.

$$
u=\arctan \left(\frac{\frac{z}{\sin (i)}}{x \cos (\Omega)+y \sin (\Omega)}\right)
$$




$$
\nu=\arctan \left(\frac{\sqrt{1-e^{2}} \sin (E)}{\cos (E)-e}\right)
$$

Finally the argument of periapsis can be found by Eq. 3.20.

$$
\omega=u-\nu
$$

\subsubsection{Perturbed Orbits}

Keplerian orbits treat the Earth as a perfectly uniform sphere, and so it acts as a point mass. This provides a good first approximation for spacecraft motion. However, the distribution of mass within the Earth is not uniform. The Earth is also not the only gravitational body either, with spacecraft seeing gravitational attraction from every planet and satellite, most notable of which is that from the Sun and Moon. Spacecraft orbiting the Earth, also face an acceleration due to the drag induced by the resistance of the Earth's upper atmosphere. Solar radiation even imparts an acceleration due to the impulse transfered from photons being absorbed or reflected off the surface of the spacecraft $[2,25]$. These forces, and various other minor forces, require us to expand on Eq. 3.1 to include these perturbing accelerations. Therefore a spacecraft's motion can be described by Eq. 3.21, where $f$ is the perturbing accelerations $[2,25]$.

$$
\begin{gathered}
\ddot{\mathbf{r}}=-\frac{\mu \mathbf{r}}{r^{3}}+f \\
f=f_{g e o}+f_{3 B}+f_{S R P}+f_{\text {Drag }}+f_{E R P}+f_{E T}+f_{\text {rel }}+f_{\text {other }}
\end{gathered}
$$

The perturbing acceleration $f$ is described by Eq. 3.22, where $f_{g e o}$ is the acceleration due to geopotential effects due to the mass distribution of the Earth, $f_{3 B}$ is the accelerations due to the gravitational influence of the Sun and Moon, $f_{S R P}$ is the solar radiation pressure acceleration, $f_{\text {Drag }}$ is the acceleration due to atmospheric drag $[2,25]$. 


$$
f_{g e o}=\nabla U=\nabla \frac{\mu}{r} \sum_{n=0}^{\infty} \sum_{m=0}^{n} \frac{R_{e}^{n}}{r^{n}} \bar{P}_{n m}(\sin \phi)\left[\bar{C}_{n m} \cos (m \lambda)+\bar{S}_{n m} \sin (m \lambda)\right]
$$

In Eq. $3.23, U$ is the gravity potential, $\mu$ is the gravitational constant of Earth, $R_{e}$ is the Earth's equatorial radius, $r$ is the spacecraft's distance from the center of the coordinate frame, $\bar{P}_{n m}$ is the normalized associated Legendre function of degree $n$ and order $m, \lambda$ and $\phi$ are longitude and latitude respectively, and $\bar{C}_{n m}$ and $\bar{S}_{n m}$ are coefficents describing the dependence on the Earth's internal mass distribution $[2,25]$.

$$
f_{3 B}=G M\left(\frac{(\mathbf{s}-\mathbf{r})}{|\mathbf{s}-\mathbf{r}|^{3}}-\frac{\mathbf{s}}{|\mathbf{s}|^{3}}\right)
$$

In Eq. 3.24, $G$ is the gravitational constant, $M$ is the mass of the perturbing body, $\mathbf{s}$ is the geocentric location of perturbing body, and $\mathbf{r}$ is the geocentric location of the spacecraft [2, 25].

$$
f_{S R P}=-P \frac{\nu A}{m} C_{r} \mathbf{u}
$$

In Eq. $3.25, P$ is the momentum flux, $\nu$ is an eclipse factor, $A$ is the cross-sectional area of the spacecraft, $C_{R}$ is the reflectivity coefficient of the spacecraft, $\mathbf{u}$ is the unit vector pointing from satellite to the sun, and $m$ is the spacecraft's mass $[2,25]$.

$$
f_{\text {Drag }}=-\frac{C_{D} A}{2 m} \rho v_{r}^{2} \mathbf{e}_{v}
$$

In Eq. $3.26, C_{D}$ is the coefficient of drag, $A$ is the spacecraft's cross-sectional area, $\rho$ is the atmospheric density, $v_{r}$ is the velocity relative to the atmosphere, $m$ is the spacecraft's mass, and $\mathbf{e}_{v}$ is the the unit vector of the relative velocity equivalent to $\frac{\mathbf{v}_{r}}{v_{r}}$.

The accelerations $f_{g e o}, f_{3 B}, f_{S R P}$, and $f_{D r a g}$ are considered sufficient for precisely describing orbits in many situations [2]. For precision modeling, the accelerations due to Earth radiation pressure $\left(f_{E R P}\right)$, Earth tides $\left(f_{E R P}\right)$, and relativity $\left(f_{r e l}\right)$, should be considered [2] and are described in more detail in the texts $[2,25]$. The remaining term $f_{\text {other }}$ handles unmodeled accelerations. These 
unmodeled accelerations could be due to thermal effects or magnetically induced forces, and an example of one of these accelerations would be the y-bias force experienced by the GPS satellites[25]

\subsection{Global Positioning System}

The Global Positioning System, GPS, is a radionavigation system providing positioning to users on Earth or in low Earth orbit, so long as at least four GPS satellites have a line of sight. GPS is separated into three segments; space segment, control segment, and user segment[26]. The first two segments are the satellites themselves(space segment), and the management of those satellites (control segment), both of which are operated by the U.S Department of Defense (DoD). The user segment covers GPS receivers themselves.

GPS measurements come from two sources, code phase(psedorange) and the carrier phase. The measureme models for both $\operatorname{code}\left(\rho^{k}\right)$ and carrier $\left(\phi^{k}\right)$ phase observations are shown by Eq. 3.27 and Eq. 3.28. The terms in these two equations show the various sources of error in the pseudorange and carrier phase. Here $k$ is the GPS satellite the measurements are coming from, $c$ is the speed of light, $r$ is the geometric range from user to GPS satellite $\left(r=\sqrt{\left(x_{u}-x^{k}\right)^{2}+\left(y_{u}-y^{k}\right)^{2}+\left(z_{u}-z^{k}\right)^{2}}\right)$, $\delta t_{u}$ is the user clock bias, $\delta t^{k}$ is the GPS clock bias, $I^{k}$ is the errors due to the ionosphere, $T^{k}$ is the errors due to the troposphere, and $\epsilon$ is unmodeled sources of error. The carrier phase model Eq. 3.28 also contains the terms $\lambda$, the GPS signal wavelength, and $N^{k}$, which is the carrier phase integer ambiguity term [26].

$$
\begin{gathered}
\rho^{k}=r^{k}+c\left[\delta t_{u}-\delta t^{k}\right]+I_{\rho}^{k}+T_{\rho}^{k}+\epsilon_{\rho} \\
\phi^{k}=\lambda^{-1}\left[r^{k}-I_{\phi}^{k}+T_{\phi}^{k}\right]+c\left[\delta t_{u}-\delta t^{k}\right] \lambda^{-1}+N^{k}+\epsilon_{\phi}
\end{gathered}
$$

The errors due to the ionosphere drop out when using dual frequency, and if the receiver is on a spacecraft the errors due to the troposphere can be dropped. 


\subsection{Kalman Filters}

A Kalman filter is a sequential estimation algorithm [25, 2, 27], which means it uses all previous all previous state and covariance information to predict its current state. The Kalman filter consists of two steps, a time update, or prediction, and a measurement update. During the time update the state vector and covariance matrix are propagated from the previous time step to the current one using the state tranisition matrix. The Kalman gain is calculated in the measurement update which maps the residuals into a correction of the state vector and to update the covariance. This is repeated until some there are no more measurements.

\subsubsection{EKF Overview}

The extened kalman filter (EKF)reduces non-linearities to a minimum [2], and is employed in realtime orbit determination programs. During its time update step, the state is propagated by some function of the previous state and an input $f\left(x_{k-1 \mid k-1}, u_{k-1}\right)$, and the error covariance is propagated using the state transition matrix (discussed further in chapter 4). A process noise $Q$ is also applied to the error covariance [2].

$$
\begin{array}{r}
x_{k \mid k-1}=f\left(x_{k-1 \mid k-1}, u_{k-1}\right) \\
P_{k \mid k-1}=\Phi P_{k-1 \mid k-1} \Phi^{T}+Q_{k} \\
\Phi=\frac{\partial f}{\partial x}
\end{array}
$$

The measurement update of the EKF follows the equations in 3.30. The innovation residual is calculated as a function of the measurements $y_{k}$ and the computed observations $h\left(x_{k \mid k-1}\right)$, which is a function of the state. The Kalman gain $K$ is calculated as a function of the error covariance matrix $P$, and the observation sensitivity matrix $H$, which is a matrix of the partial derivatives of the computed obseravbles with respect to the state. The Kalman gain is then used to update the state and the error covariance for the current epoch [2]. 


$$
\begin{array}{r}
r_{k}=y_{k}-h\left(x_{k \mid k-1}\right) \\
K_{k}=P_{k \mid k-1} H_{k}^{T}\left(H_{k} P_{k \mid k-1} H_{k}^{T}+R_{k}\right)^{-1} \\
x_{k \mid k}=x_{k \mid k-1}+K r_{k} \\
P_{k \mid k}=\left(I-K_{k} H_{k}\right) P_{k \mid k-1} \\
H_{k}=\frac{\partial h}{\partial x}
\end{array}
$$

The EKF will repeat these steps until there are no more measurements made. While the EKF improves performance it also requires more computational resources.

\subsubsection{Equations for Reduced Dynamic POD EKF}

These equations correspond to the reduced dynamic section of chapter $2[12,5]$.

The time update of the Kalman filter is as follows $[12,5]$.

$$
\begin{gathered}
\tilde{\mathbf{X}}_{j+1}=\Phi_{j} \hat{\mathbf{X}}_{j}+B w_{j} \\
\tilde{P}_{j+1}=\Phi_{j} \hat{P}_{j} \Phi_{j}^{T}+B w_{j} B^{T} \\
B=\left[\begin{array}{c}
0 \\
I_{p}
\end{array}\right]
\end{gathered}
$$

In Eqs. 3.31 through 3.33, $I_{p}$ is a unit vector, $w_{j}$ is a white noise process of covariance $Q_{j}$ which is assumed diagonal with elements $q_{i}=\left(1-m_{i}^{2}\right) \sigma_{i}^{2}$, where $m_{i}$ is defined by Eq. 3.35 and $\sigma_{i}$ is the steady-state uncertainty $[12,5]$.

$$
\Phi_{j}=\left[\begin{array}{cc}
\Phi_{x}(j+1, j) & \Phi_{x p}(j+1, j) \\
0 & M_{j}
\end{array}\right]
$$




$$
m_{i}=e^{\frac{-\left(t_{j+1}-t_{j}\right)}{\tau_{i}}}
$$

Here in Eq. $3.34 \Phi_{x p}$ relates $\tilde{\mathbf{X}}_{j+1}$ to $\mathbf{p}_{j}$ and $M_{j}$ is $3 x 3$ diagonal matrix with its nonzero elements represented by Eq. 3.35, where $\tau$ is the correlation time constant. Relative weighting of the dynamic model is varied by selecting different values for $\sigma$ and $\tau$. Increasing $\tau$ and decreasing $\sigma$ drive the reduced dynamic approach to dynamic tracking, and doing the inverse drives it to a purely kinematic tracking. The measurement update of the Kalman filter remains the same with the exception that the state and error covariance matrix are associated with the fictitious force as well as the spacecraft state $[12,5]$. 


\section{Chapter 4}

\section{Technical Approach}

The proposed solution for POD of spacecraft using duty cycled GPS receivers, is a simplified reduced dynamic estimator. This approach to reduced dynamic POD differs from previous work in that it uses two-body dynamics to propagate the state of the spacecraft, as well as using process noise on the spacecraft's velocity and position instead of using empirical forces. The two-body reduced dynamic (TBRD) approach reduces the computational load, which would be beneficial applied in real-time to a spacecraft with limited computational resources, such as a CubeSat. This approach was implemented in Matlab and tested using simulated data.

This chapter discusses in detail how this simplified reduced dynamic approach is implemented breaking down in detail the Kalman filter used used, and how the state transition matrix is formulated.

\subsection{Algorithm Overview}

GPS measurements are combined with a two-body dynamic model in an error state extended kalman filter. While the GPS receiver is off the dynamic force model is used to propagate the position and velocity states. The force model is also used to formulate the state transition matrix. When the receiver is turned on and at least four GPS satellites are being tracked, prior to the first measurement update, a linear least square estimator is used to correct for position errors that were accrued during the GPS off cycle. After the linear least squares fixing, the EKF proceeds through the measurement update. 


\subsection{State Transition Matrix Formulation}

The state transition matrix $(\mathrm{STM})$ takes the $\operatorname{state}(\mathbf{Y}=[\mathbf{r}, \mathbf{v}])$ and error covariance from the previous epoch's estimation to the current epoch's prediction and can be described by Eq. 4.1, or as a matrix of the partial derivative of the state $\mathbf{Y}$ with respect to the previous state $\mathbf{Y}_{0}$ [2] (see Eqs. 3.29, 3.31, $3.29)$.

$$
\Phi\left(t, t_{0}\right)=\frac{\partial \mathbf{Y}(t)}{\partial \mathbf{Y}\left(t_{0}\right)}
$$

\subsubsection{Two-Body State Transition Matrix}

The state transition matrix for the TBRD approach is the product of the orbital elements transition

matrix $\Phi_{\alpha}$ and the partials of the spacecraft state with respect to the orbital elements $\frac{\partial \mathbf{Y}}{\partial \boldsymbol{\alpha}}[2]$. Only the mean anomaly changes with time and the rest of the orbital elements in the vector $\boldsymbol{\alpha}=$ $(a, e, i, \Omega, \omega, M)$ remain constant in a two-body model, resulting in the matrix shown in Eq. 4.2. The mean anomaly is a function of the mean motion, which itself is a function of the semi-major axis, with the resulting partial as the only off diagonal element in the matrix calculated by Eq. 4.3.

$$
\Phi_{\text {alpha }}\left(t, t_{0}\right)=\left[\begin{array}{cccccc}
1 & 0 & 0 & 0 & 0 & 0 \\
0 & 1 & 0 & 0 & 0 & 0 \\
0 & 0 & 1 & 0 & 0 & 0 \\
0 & 0 & 0 & 1 & 0 & 0 \\
0 & 0 & 0 & 0 & 1 & 0 \\
\frac{\partial M(t)}{\partial a\left(t_{0}\right)} & 0 & 0 & 0 & 0 & 1
\end{array}\right]
$$

To obtain partials of the spacecraft state with respect to the orbital elements $\frac{\partial \mathbf{Y}}{\partial \boldsymbol{\alpha}}$, the perifocal coordinates are found using Eqs. 3.6 and 3.9, and the partials of the Gaussian vectors with respect to $i, \Omega$, and $\omega$. The resulting $\frac{\partial \mathbf{Y}}{\partial \boldsymbol{\alpha}}$ is shown in Eq. 4.4. 


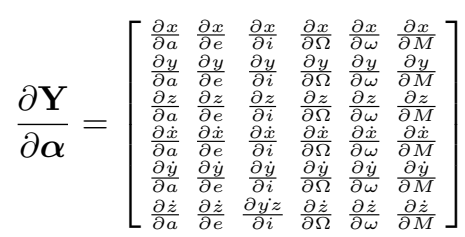

The partial derivatives for the previous epoch are then inverted to become the partial derivative of the Keplerian elements with respect to the state(Eq. 4.5). This is done using Poisson Parentheses [2], which are shown in Eq. 4.6. The state transition matrix is then the result Eq.(4.7).

$$
\begin{gathered}
\frac{\partial \mathbf{Y}}{\partial \boldsymbol{\alpha}}^{-1}=P(\boldsymbol{\alpha}, \boldsymbol{\alpha})\left(\left(\frac{\partial \mathbf{v}}{\partial \boldsymbol{\alpha}}\right),\left(-\frac{\partial \mathbf{r}}{\partial \boldsymbol{\alpha}}\right)\right) \\
P(a, M)=\frac{-2}{n a} \\
P(e, \omega)=\frac{\sqrt{1-e^{2}}}{n a^{2} e} \\
P(e, M)=\frac{-\left(1-e^{2}\right)}{n a^{2} e} \\
P(i, \Omega)=\frac{1}{n a^{2} \sqrt{1-e^{2}} \sin (i)} \\
P(i, \omega)=\frac{-\cot (i)}{n a^{2} \sqrt{1-e^{2}}} \\
\Phi\left(t, t_{0}\right)=\frac{\partial(Y)}{\partial(A)} * \frac{\partial\left(A_{0}\right)}{\partial\left(Y_{0}\right)}
\end{gathered}
$$

\subsection{POD EKF}

The EKF used in this work is an error state filter, which solves for the difference of the position and velocity states with respect to an assumed nominal. The state vector is thus represented by Eq. 4.8, where $c \delta t$ and $c \delta \dot{t}$ are the clock bias and clock drift respectively and $N_{k \times 1}$ (k is the number of satellites at the current epoch) is the phase ambiguity for each GPS satellite being tracked. 


$$
\mathbf{x}=\left[\begin{array}{c}
\Delta x \\
\Delta y \\
\Delta z \\
\Delta \dot{x} \\
\Delta \dot{y} \\
\Delta \dot{z} \\
c \delta t \\
c \delta \dot{t} \\
N_{k \times 1}
\end{array}\right]
$$

\subsubsection{Assumptions}

For this work, the total duty cycle is assumed to be 10 minutes, and various percentages of on-time are investigated. To initialize the EKF, the duty cycle percentage was used to specify the amount of assumed error, as both the total cycle time (on and off), and the on time. To emulate realistic tracking of satellites when the receiver is turned back on, the number of GPS satellites being tracked is increased depending on visibility of the satellite and an empirical probability that was determined from analyzing the rate that satellites were acquired in hardware in the loop testing of the NovAtel receiver that will fly on STF-1.

\subsubsection{Prediction}

During the predicition step, or time update, the spacecraft's position and velocity are updated using the dynamic model. When the new state is calculated, the state transition matrix is formulated following the process discussed in the previous section. The newly formulated state transition matrix is used to propagate the error covariance matrix, however the error states are left as zero.

When a phase break occurs (e.g., when the reciever is duty cycled), either from a data editor [28] or after a duty off cycle, a white-noise reset occurs on the associated carrier-phase ambiguity parameter. This is done by resetting the a priori estimated phase bias to the difference of the ionospheric free range and phase observables, increasing the error covariance of the phase ambiguities to $100^{2}$ cycles, and setting all cross-correlations with the ambiguity and all other filter parameters to zero [1]. 


\subsubsection{Measurement Update}

The measurement update is only performed when the GPS receiver is on and the receiver is tracking at least four GPS satellites. When at least four GPS satellites are tracked, prior to the first EKF measurement update of the duty on cycle, the spacecraft position, computed pseudorange, and error covariance matrix are updated using a linear least squares (LLS) estimator that is iterated until convergence, correcting for drift accumulated while the GPS is off.

The observation sensitivity matrix $(H)$, shown in Eq.(4.9), is used in the measurement update, here the $1_{u}^{i}$ represents the unit vector from the user's nominal position, $u$, to each GPS satellite, $i$, and the the $\mathbf{I}$ is the identity matrix for the phase bias parameters.

$$
H=\left[\begin{array}{ccccc}
\mathbf{1}_{\mathbf{u}}^{\mathbf{1}} & 0_{1 x 3} & 1 & 0 & 0_{1 x k} \\
\vdots & \vdots & \vdots & \vdots & \vdots \\
\mathbf{1}_{\mathbf{u}}^{\mathbf{k}} & 0_{1 x 3} & 1 & 0 & 0_{1 x k} \\
\mathbf{1}_{\mathbf{u}}^{\mathbf{1}} & 0_{1 x 3} & 1 & 0 & \\
\vdots & \vdots & \vdots & \vdots & I_{k x k} \\
\mathbf{1}_{\mathbf{u}}^{\mathbf{k}} & 0_{1 x 3} & 1 & 0 &
\end{array}\right]
$$

The measurement vector shown in Eq.(4.10) contains the observed pseudoranges $\left(\rho^{k}\right)$ and carrier phase $\left(\phi^{k}\right)$ measurements from each satellite being tracked by the receiver.

$$
Z=\left[\begin{array}{c}
\rho^{1} \\
\vdots \\
\rho^{k} \\
\phi^{1} \\
\vdots \\
\phi^{k}
\end{array}\right]
$$




\section{Chapter 5}

\section{Simulation Setup}

All data used in testing for this thesis is simulated in Matlab.

\subsection{Orbit Selection}

Investigating multiple different orbits allows the investigation on the impact of orbit selection on the performance of this approach. Ten different LEO orbits were chosen to be investigated for this work as listed in Table $5.1[29,30]$. The orbits were chosen for their variation in inclination and semi-major axis. The eccentricities of most of the orbits are nearly circular, but the differential equations of motion can not be solved with a 0 eccentricity, thus the eccentricity for those were given a small value of 0.0005 . The second entry in Table 5.1 is listed as ISS, as many CubeSats are brought to the ISS to be deployed, and thus share very similar orbital characteristics. It should also be noted, that, for simplicity, for all but the ISS orbit, the initial right ascension of the ascending node, and the initial argument of perigee are held to be 0 . 
Table 5.1: Investigated Satellite Orbital Parameters

\begin{tabular}{rrrr} 
Satellite & Semi-major Axis $(\mathbf{k m})$ & Eccentricity & Inclination(degrees) \\
\hline STF-1 & 6878.14 & 0.006 & 85 \\
ISS & 6775 & 0.0006 & 51.64 \\
RAVAN XB3 & 6988 & 0.0005 & 98 \\
Athenoxat-1 & 6921 & 0.0005 & 15 \\
SNaP-3 A & 7018.1 & 0.0228 & 64.8 \\
Lemur-2 2 & 7026 & 0.0005 & 6 \\
SMDC-ONE 3.1 & 70531.1 & 0.0295 & 120.5 \\
HawkSat 1 & 6821 & 0.0005 & 40.4 \\
NanoSail-D2 & 7021 & 0.0005 & 72 \\
Perseus 000 & 6664.6 & 0.002 & 34.5
\end{tabular}

\subsection{Low Earth Orbit Simulation}

For each of the ten selected orbits, the position and velocity in the Earth centered inertial (ECI) frame are calculated for every tenth of a second of a 3 hour observation period, starting at noon on January 1, 2017. A direction cosine matrix (DCM) is created for each position as well, to rotate the position and velocity in ECI to an Earth centered, Earth fixed (ECEF) frame for generation of the GPS observables. The DCM includes compensation for the leap seconds between International Atomic Time (TAI), and Coordinated Universal Time (UTC). Generation of this orbit data takes into account accelerations due to the Earth's harmonic gravity field $\left(f_{\text {geo }}\right)$ Eq. (3.23) and the perturbing gravitational effects of the Sun and $\operatorname{Moon}\left(f_{3 B}\right)$ Eq. (3.24) [2].

Using methods to solve the differential equations of motion from Montenbruck [2], the orbit generator is given the initial Keplerian elements, which is then used to solve for the initial position and velocity of the spacecraft. From the initial position and velocity, the differential equations of motion and perturbing accelerations are solved at each epoch, which for these tests corresponded to the GPS receiver measurement rate of $10 \mathrm{~Hz}$.

Another orbit was simulated for STF-1 that includes accelerations due to $\operatorname{drag}\left(f_{\text {Drag }}\right.$ Eq. 3.26) and solar radiation pressure $\left(f_{S R P}\right.$ Eq. 3.25). To calculate these accelerations STF-1 was assumed to have a $C_{D}$ of 2.2 , a cross-sectional area (A) of $0.03 m^{2}$, and a $C_{R}$ of 1 . This orbit is not included in the sensitivity study, and is discussed separately in the results chapter.

\subsection{GPS Observable Simulation}

For each satellite, $10 \mathrm{~Hz}$ GPS observables are simulated for a 3 hour period. While generating the GPS observables the spacecraft is currently treated as just a receiver moving through space, 
not account for the spacecraft's attitude, and thus errors imposed by the spacecraft (i.e., occlusion of signals, etc) are ignored. Error due to the ionosphere is included in the generated observables. Unlike a GPS receiver on the ground, a spacecraft in LEO can see satellites that are orbiting on the opposite side of the Earth, with limitations. An elevation angle (i.e., angle from receiver to GPS satellite, with respect to a local flat plane at the receiver) mask is used to determine how far behind the Earth the receiver can track satellites. The equation to calculate this angle is shown below, where $\mathrm{h}$ is the altitude from the surface of Earth.

$$
\theta=\arccos \left(R_{\text {earth }} / R_{\text {earth }}+h\right)
$$

Using MATLAB functions from the SatNav Toolbox [31], the dual frequency GPS observables (code range, carrier phase, satellite PRN numbers, etc.) for each position of the orbit are generated. After generating the GPS observables the effects of duty cycling is applied by inserting a random integer to the carrier phase observables to represent the change in carrier phase integer ambiguity every 10 minutes (corresponding to the time when the receiver will be said to be on). A flag is signifying that this phase break has taken place is also put at the beginning of each 10 minute interval, which will signal the EKF to reset the current estimated state of the phase ambiguities.

\subsection{Hardware in the Loop Testing}

During the Summer of 2016, a Spirent GPS simulator was provided by the NASA IV\&V STF-1 CubeSat team for hardware in the loop (HIL) tests with the flight GPS receiver. The simulator was set up to provide single frequency observables, for a spacecraft with the orbital parameters for STF-1 (as can be seen in Table 1), over a 3 hour period at $10 \mathrm{~Hz}$. The test was run without duty cycling the receiver to collect a baseline, then different duty cycles were investigated from a $10 \%$ on time to $90 \%$ on time. The collected data was processed using GIPSY[32] to get the positioning of the spacecraft and the satellites tracked at each epoch. This hardware in the loop testing helped provide an estimate of how the satellites were added in, allowing the Matlab simulation to be more realistic, see Figures 5.1 and 5.2. Without this estimate, the receiver automatically started tracking every satellite that was within line of sight. This estimate was implemented in the Matlab code as a function to gradually add in satellites being tracked, so long as they were in line of sight. 


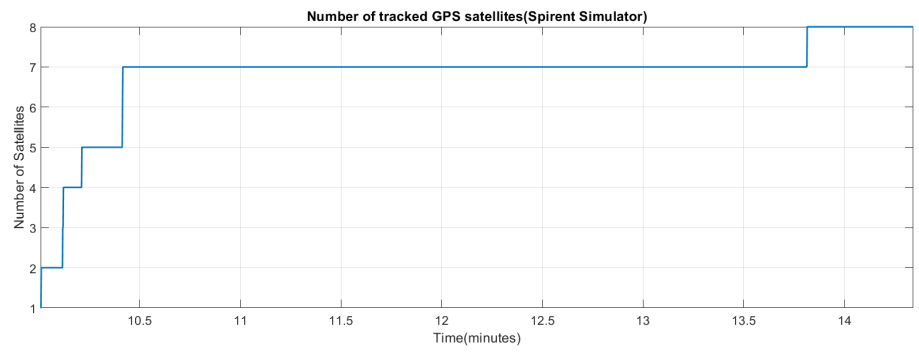

Figure 5.1: Number of satellites over one on cycle using the Spirent GPS simulator.

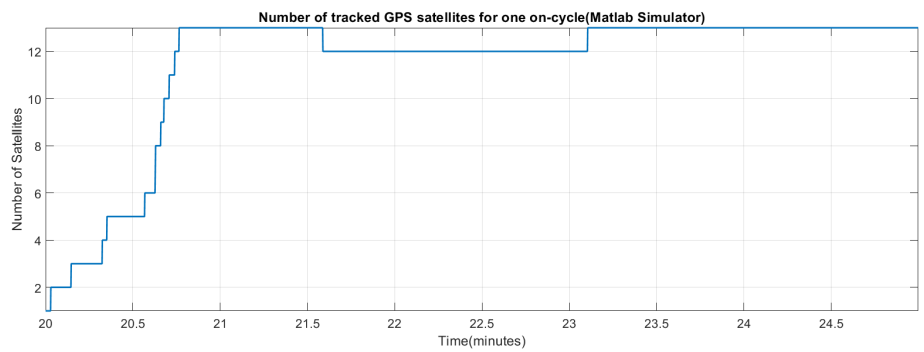

Figure 5.2: Number of satellites over one on cycle in Matlab simulation.

\subsection{Sensitivity Study Design}

A sensitivity study was set up to compare the results of the EKF for both the kinematic and TBRD approaches, as well as the change in duty cycle on time (in 10\% increments of the 10 minute total duty cycle). For each set of inputs the EKF is ran 5 times. This is due to the randomness of adding satellites as discussed in the initialization section above, therefore each run of the EKF will produce varying results with the same inputs. The input parameters for the EKF that were changed in the sensitivity study are shown in Table 5.2.

Table 5.2: Sensitivity Study, EKF Input Parameters

\begin{tabular}{rr} 
Input Parameter & Parameter Values \\
\hline Satellite Orbit/Observables & See Table 5.1 \\
STM & Kinematic or TBRD \\
Duty Cycle Percentage & $10 \%-100 \%(10 \%$ increments $)$ \\
MC Iteration & $1-5$
\end{tabular}




\section{Chapter 6}

\section{Results}

A comparison was made between the TBRD approach and a purely kinematic approach, to demonstrate the improvement, of the TBRD approach. The following sections will discuss the results of using both the kinematic and TBRD approaches separately, then the comparison between the two will be discussed. For the results, only the time that the GPS is on will be considered as the use of any relevant dynamic model will inherently out-perform a kinematic estimator while the GPS is off.

Each POD approach is initialized in the EKF differently. The selection of an approach, as well as the specified duty cycle determine the process noise for the position and velocity states as shown in Eq. (6.4), with $t_{\text {on }}$ being the time the GPS is on during the duty cycle.

$$
\begin{gathered}
P=\left[\begin{array}{cccc}
I_{6 x 6} & 0_{6 x 1} & 0_{6 x 1} & 0_{8 x n_{\text {sat }}} \\
0_{1 x 6} & 10 e^{-3} & 0 & \vdots \\
0_{1 x 7} & \ldots & 1 e^{-5} & \vdots \\
0_{n_{\text {sat } x 8}} & \ldots & \ldots & I_{n_{\text {sat }} n_{\text {sat }}}
\end{array}\right] \\
Q_{\text {pos }}=I_{3 x 3} *\left(\frac{K}{t_{\text {on }} 600}\right)^{2} \\
Q_{v e l}=I_{3 x 3} *\left(\frac{1}{t_{\text {on }} 600}\right)^{2}
\end{gathered}
$$




$$
\left[\begin{array}{ccccc}
Q_{\text {pos }} & \ldots & \ldots & \ldots & 0 \\
\vdots & Q_{v e l} & & & \vdots \\
\vdots & & 200 & & \vdots \\
\vdots & & & 20 & \vdots \\
0 & \ldots & \ldots & \ldots & 0_{n_{s a t} x n_{s a t}}
\end{array}\right]
$$

The value of $K$ has been determined by finding the average integration error for a $50 \%$ duty cycle ( $K=500 \mathrm{~m}$ for the TBRD approach and $K=1 e^{6} \mathrm{~m}$ for the kinematic).

\subsection{Kinematic}

As a demonstration of the impact of duty cycling on GPS based POD, as well as a baseline to compare the TBRD approach to, a kinematic estimator. This essentially eliminates the time update step of the EKF, relying solely on the measurement update to estimate the state. The mean and median of the mean root mean squares error (RMSE) values for all ten orbits being investigated are shown in Figure 6.1.

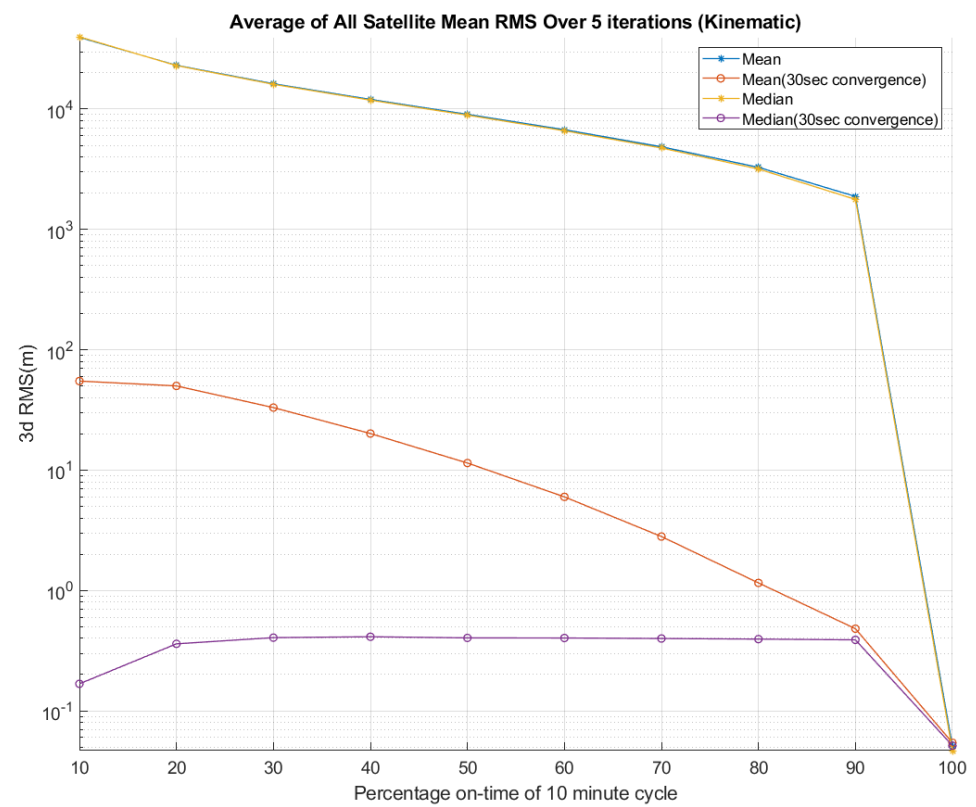

Figure 6.1: RMS Errors for various duty cycle times for pure kinematic approach

As can be seen in Figure 6.1, and Table 6.1, the mean RMS is significantly higher than the 
Table 6.1: Root Mean Square Error across all 10 Satellites for 10\% increments of a 10 Minute Duty Cycle, when GPS is on, Kinematic estimator

\begin{tabular}{r|rrr|rrr|} 
DC On\% & \multicolumn{3}{|c|}{ Total On Cycle } & \multicolumn{3}{c|}{ Post 30s Convergence } \\
\hline & Mean (m) & Median $(\mathbf{m})$ & Max $(\mathbf{m})$ & Mean $(\mathbf{m})$ & Median $(\mathbf{m})$ & Max (m) \\
\hline $10 \%$ & 39457 & 2054.5 & 378500 & 54.95 & 0.1675 & 547.8 \\
$20 \%$ & 23136 & 1344.2 & 220860 & 50.09 & 0.3600 & 498.1 \\
$30 \%$ & 16251 & 1072.2 & 154130 & 33.09 & 0.4054 & 327.7 \\
$40 \%$ & 12040 & 918.08 & 113230 & 20.13 & 0.4123 & 198.0 \\
$50 \%$ & 9049.7 & 815.71 & 84126 & 11.46 & 0.4034 & 111.2 \\
$60 \%$ & 6743.2 & 741.38 & 61642 & 5.993 & 0.4026 & 56.59 \\
$70 \%$ & 4867.4 & 684.25 & 43330 & 2.806 & 0.3987 & 24.77 \\
$80 \%$ & 3282.9 & 638.57 & 27842 & 1.156 & 0.3942 & 8.313 \\
$90 \%$ & 1881.9 & 600.97 & 14371 & 0.4809 & 0.3886 & 1.615 \\
$100 \%$ & 0.0507 & 0.0484 & 0.0718 & 0.0542 & 0.0513 & 0.0731
\end{tabular}

median. The skew of the mean kinematic RMSE values can be explained by the fact that satellite 8 (see Table 5.1 and Figure 6.1) suffers from larger error values, even when compared to similar orbits. The cause of these larger errors was found to be due to poor geometry. The poor geometry result in a higher value of DOP, delaying the measurement update, resulting in a worse initial condition for the EKF to update.

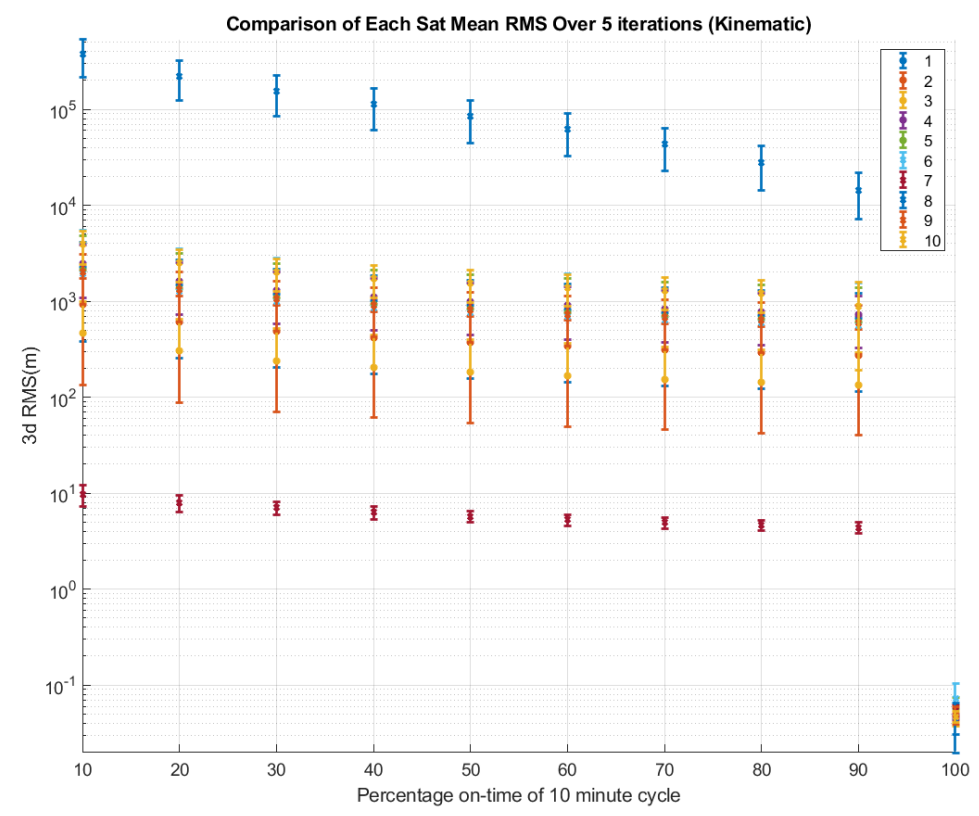

Figure 6.2: Mean RMS Errors for various duty cycle times for pure kinematic approach

It can be seen in Figure 6.1 that satellite 7 has lower than average kinematic errors, though the reason for this lower error is unknown and not seen in the TBRD approach. 


\subsection{TBRD}

The mean and median of the mean root mean squares error (RMSE) values for all ten orbits being investigated are shown in Figure 6.3. It can be observed that the mean RMSE for a $70 \%$ duty cycle in the TBRD approach spikes to a higher value when considering the data after 30 seconds of convergence.

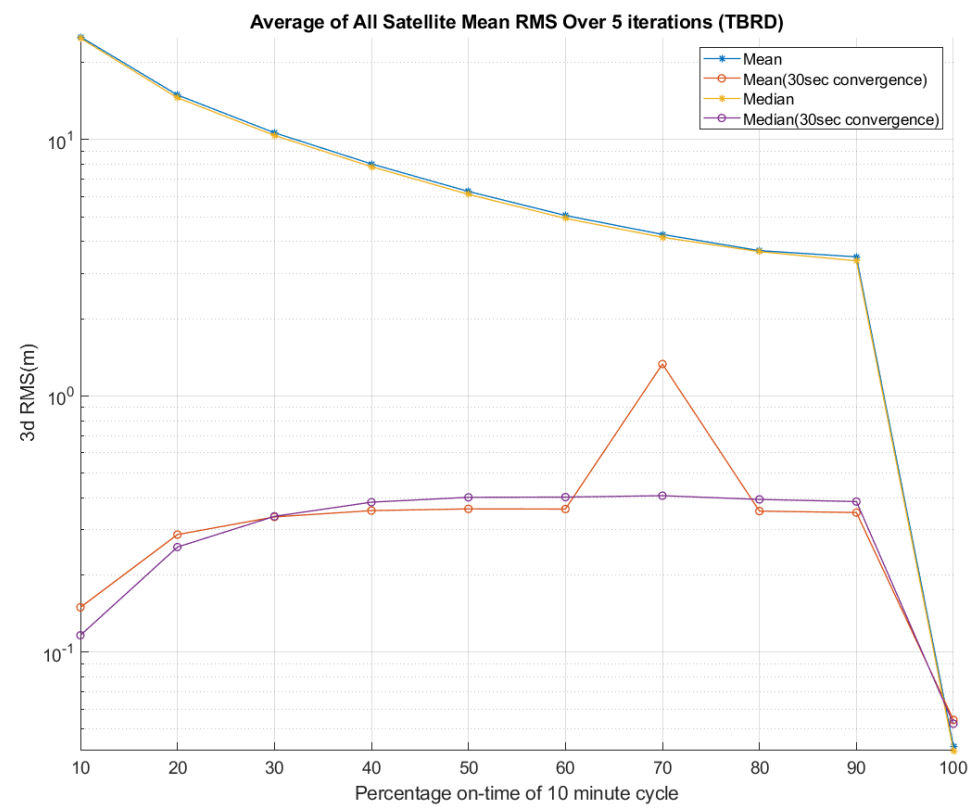

Figure 6.3: RMS Errors for various duty cycle times for TBRD approach

Table 6.2: Root Mean Square Error across all 10 Satellites for $10 \%$ increments of a 10 Minute Duty Cycle, when GPS is on, TBRD estimator

\begin{tabular}{r|rrr|cr|r|} 
DC On\% & \multicolumn{3}{|c|}{ Total On Cycle } & \multicolumn{3}{c|}{ Post 30s Convergence } \\
\hline & Mean (m) & Median $(\mathbf{m})$ & Max $(\mathbf{m})$ & Mean $(\mathbf{m})$ & Median $(\mathbf{m})$ & Max (m) \\
\hline $10 \%$ & 25.19 & 8.636 & 171.2 & 0.1495 & 0.1162 & 0.2730 \\
$20 \%$ & 14.93 & 6.940 & 90.38 & 0.2874 & 0.2569 & 0.4789 \\
$30 \%$ & 10.63 & 5.997 & 56.65 & 0.3368 & 0.3385 & 0.5212 \\
$40 \%$ & 8.0362 & 5.277 & 36.56 & 0.3564 & 0.3845 & 0.5265 \\
$50 \%$ & 6.274 & 4.740 & 23.22 & 0.3620 & 0.4017 & 0.5195 \\
$60 \%$ & 5.057 & 4.332 & 14.32 & 0.3615 & 0.4025 & 0.5091 \\
$70 \%$ & 4.266 & 4.013 & 8.806 & 1.3304 & 0.4076 & 10.0174 \\
$80 \%$ & 3.688 & 3.762 & 5.270 & 0.3549 & 0.3941 & 0.4868 \\
$90 \%$ & 3.486 & 3.539 & 5.169 & 0.3503 & 0.3866 & 0.4790 \\
$100 \%$ & 0.0429 & 0.0428 & 0.0532 & 0.0543 & 0.0525 & 0.0750
\end{tabular}

Examining Figure 6.4, the culprit for this spike appears to be satellite 4 (see Table 5.1). This spike in error is only observed when considering errors after the filter has converged for 30 seconds. 


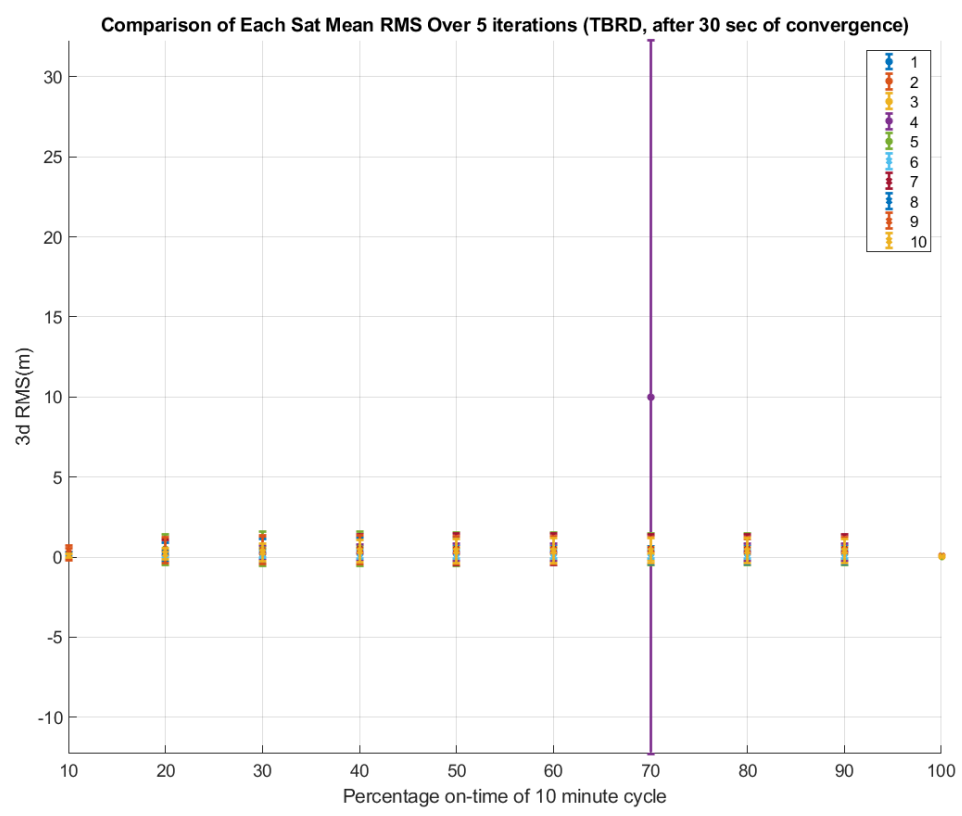

Figure 6.4: Mean RMS Errors for various duty cycle times for the TBRD approach after 30 seconds of convergence.

As with the skew of the errors for satellite 8, which can still be observed, though significantly lower, in Figure 6.5, the cause of this spike was found to be poor observation geometry, at that particular duty cycle percentage.

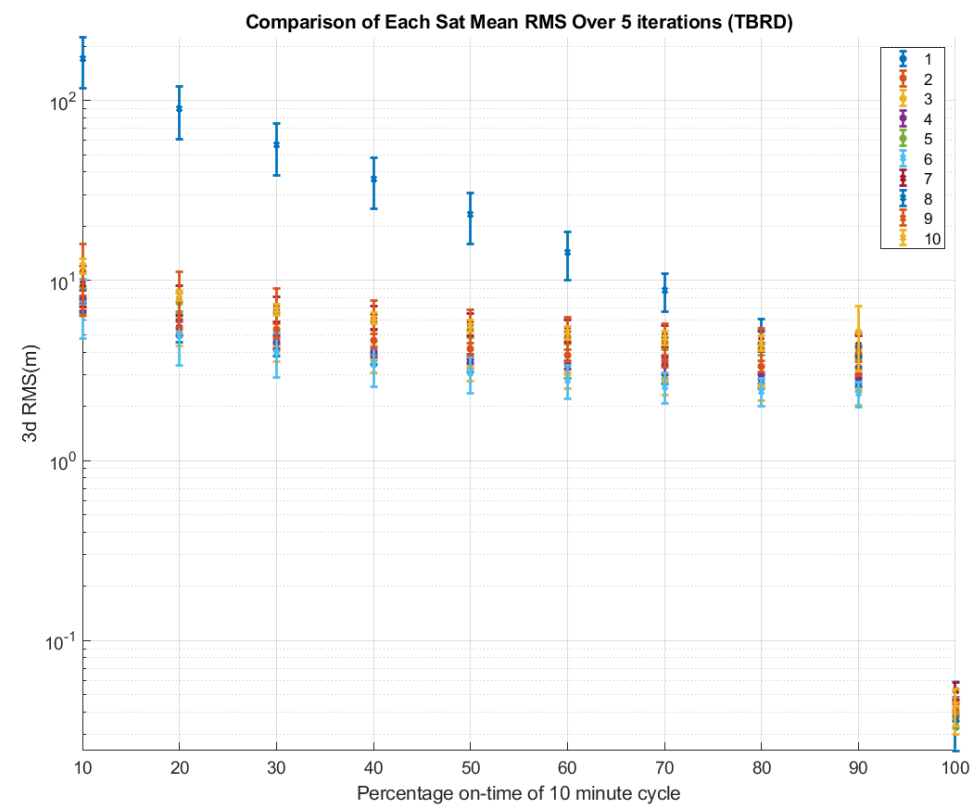

Figure 6.5: Mean RMS Errors for various duty cycle times for the TBRD approach. 


\subsection{Comparison}

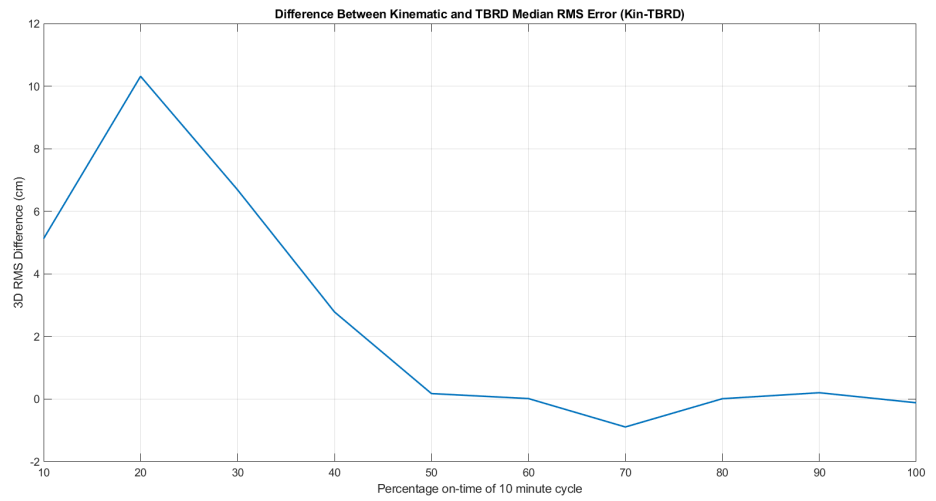

Figure 6.6: Difference of median RMS errors for various duty cycle times for the TBRD approach after 30 seconds of convergence.

For a fair comparison between the kinematic and TBRD approaches only the median RMSE after 30 seconds of convergence is considered. Prior to the convergence the difference is extremely evident, as the kinematic estimator takes longer to converge and provide good solutions. From Table 6.3 and Figure 6.6, we can see that the TBRD approach provides more accurate solution compared to a purely kinematic estimator. The exception to this is during the $70 \%$ duty cycle where the TBRD sees a spike in RMSE due to one satellite's larger error.

Table 6.3: Difference between kinematic and TBRD median RMSE after 30 seconds of convergence.

\begin{tabular}{|r|r|}
\hline DC On\% & $\Delta$ RMSE Median \\
\hline 10 & 0.0513 \\
20 & 0.1031 \\
30 & 0.0669 \\
40 & 0.0278 \\
50 & 0.0018 \\
60 & 0.0002 \\
70 & -0.0089 \\
80 & 0.0001 \\
90 & 0.0020 \\
100 & -0.0012 \\
\hline
\end{tabular}

\subsection{STF-1 Orbit with Drag and Solar Radiation Pressure Perturbations}

This orbit did not go through the five sensitivity study iterations, instead only going through 1 iteration for the different duty cycle percentages. Both the kinematic and TBRD approaches were 
investigated, as well as a full gravity model, including the perturbing accelerations due to the Earth's oblateness, to propagate the spacecraft state and error covariance. From Figure 6.7 (see Table 6.4 for the values associated with plots), it can be seen that the full gravity model performs marginally better than the TBRD approach, and both approaches greatly outperform the kinematic estimation. At $20 \%$ duty cycle the RMSE for the full gravity approach spikes to extremely high value for an unknown reason. Looking in to the cause, the DOP is extremely high(up to $10^{8}$ ) during this run, and bad satellite geometry would seem to be the culprit. However if that were the case, the impact of that poor geometry would be present at all duty cycle percentages. Where the full gravity model propagation really benefits the POD method is during the GPS off-cycles. In Figure 6.8, it is clear that the full gravity model integration error while the GPS is off remains under 10 meters, while the two-body approaches $1000 \mathrm{~m}(1 \mathrm{~km})$ errors at a $50 \%$ duty cycle. An alternate approach was

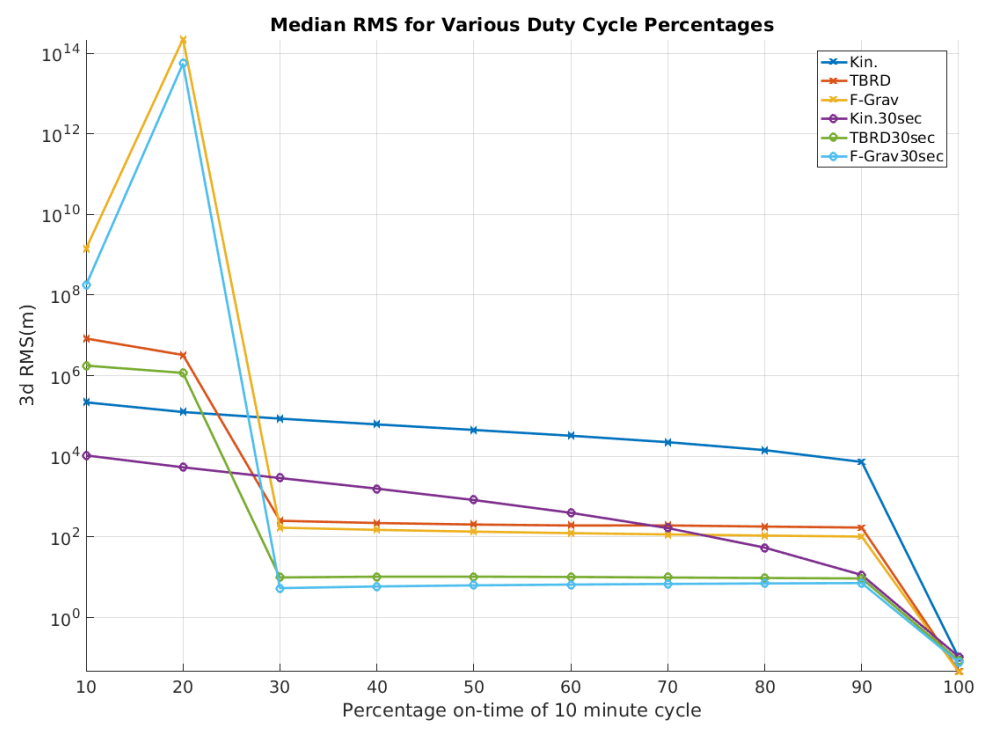

Figure 6.7: RMS Errors for various duty cycle times for kinematic, TBRD, and full gravity approach

investigate for a $50 \%$ duty cycle, where the two-body dynamic model is used to formulate the state transition matrix to propagate the error-covariance, while a a dynamic model that includes the perturbing accelerations due to the Earth's oblateness is used to propagate the spacecraft's position and velocity. While it performs about the same as the only full gravity approach, as seen in Figure 6.9, it does provide some benefit to the integration error, which can be observed in Figure 6.8. 
Table 6.4: Root Mean Square Error for POD when using STF-1 orbit with drag and solar radiation pressure perturbations when GPS is on.

\begin{tabular}{rrrr|rrr|} 
DC On\% & \multicolumn{3}{|c|}{ Total On Cycle } & \multicolumn{3}{c|}{ Post 30s Convergence } \\
\hline & Kin $(\mathbf{m})$ & TBRD $(\mathbf{m})$ & Full Grav $(\mathbf{m})$ & Kin $(\mathbf{m})$ & TBRD $(\mathbf{m})$ & Full Grav (m) \\
\hline $10 \%$ & $2.1812 \mathrm{e} 5$ & $8.3477 \mathrm{e} 6$ & $1.4147 \mathrm{e} 9$ & $1.0517 \mathrm{e} 4$ & $1.7794 \mathrm{e} 6$ & $1.7576 \mathrm{e} 8$ \\
$20 \%$ & $1.2559 \mathrm{e} 5$ & $3.2660 \mathrm{e} 6$ & $2.2386 \mathrm{e} 14$ & 0.2874 & 0.2569 & \\
$30 \%$ & 85983 & 251.64 & 169.59 & 2889.6 & 9.8823 & 5.3670 \\
$40 \%$ & 61889 & 221.38 & 149.81 & 1574.7 & 10.328 & 5.9170 \\
$50 \%$ & 45059 & 202.94 & 135.33 & 823.59 & 10.337 & 6.2939 \\
$60 \%$ & 32409 & 192.13 & 124.15 & 396.19 & 10.164 & 6.5839 \\
$70 \%$ & 22430 & 192.85 & 115.30 & 165.55 & 9.8542 & 6.8200 \\
$80 \%$ & 14238 & 180.84 & 108.08 & 54.313 & 9.5841 & 7.0142 \\
$90 \%$ & 7261.2 & 170.82 & 102.07 & 11.398 & 9.3323 & 7.1717 \\
$100 \%$ & 0.1006 & 0.0473 & 0.0455 & 0.1068 & 0.0861 & 0.0773
\end{tabular}

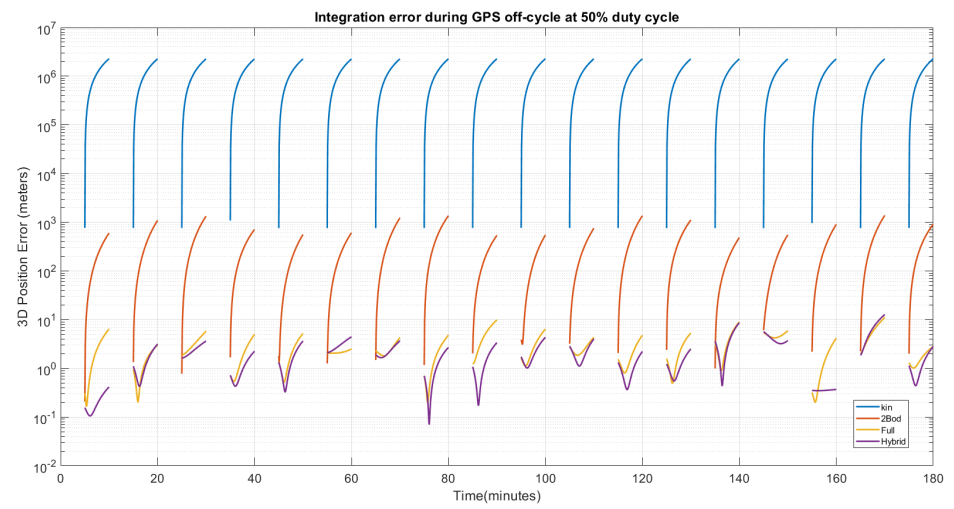

Figure 6.8: 3D position integration error for all investigated POD approaches during GPS off-cycles at $50 \%$ duty cycle.

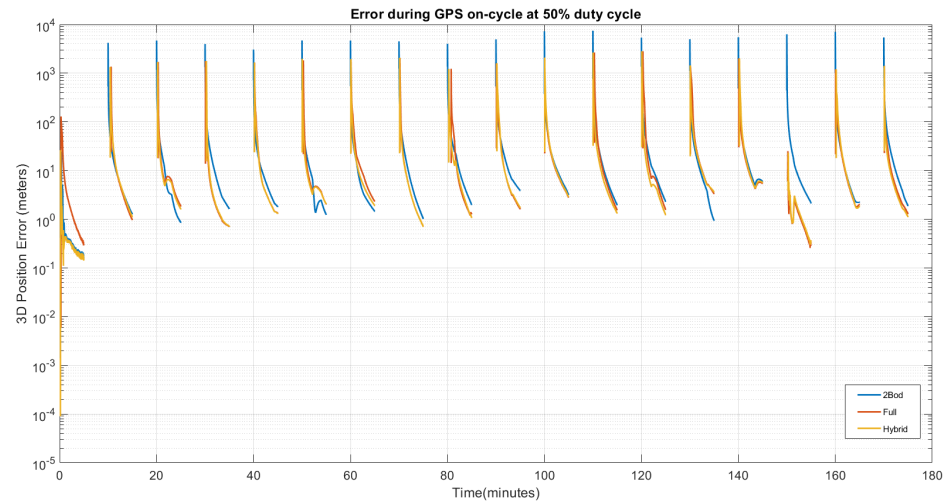

Figure 6.9: 3D position Error for all except the kinematic POD approaches during GPS on-cycles at $50 \%$ duty cycle. 


\section{Chapter 7}

\section{Conclusions \& Future Work}

\subsection{Conclusions}

Simulated orbits and GPS observables was simulated for ten different CubeSats. The GPS observables were altered to represent power duty cycled data and a simplified reduced dynamic approach was investigated. The approach, uses a two-body dynamic model to propagate the spacecraft state while GPS is off, and provides a state prediction in an extended Kalman filter. This was compared to a kinematic approach where the state estimation relied only on GPS observations.

The TBRD approach has proven to be beneficial when the GPS receiver must be power duty cycled. The TBRD approach provides faster convergence times, and marginally less position error post convergence, over the kinematic approach.

The TBRD approach could be of benefit to small satellites that need to have precise positioning, but cannot afford the resources to leave a GNSS receiver on continuously.

\subsection{Future Work}

STF-1 will be launched some time in 2018, and GPS data collected on orbit will be used to validate this approach during post- processing. It would be interesting to attempt to apply the TBRD approach for real time POD on STF-1. 


\section{Bibliography}

[1] Lantto, S. and Gross, J. N., "Precise Orbit Determination Using Duty Cycled GPS Observations," 2018 AIAA Modeling and Simulation Technologies Conference, 2018, p. 1393.

[2] Montenbruck, O. and Gill, E., "Satellite orbits," Springer, Vol. 2, 2000, pp. 257-291.

[3] Tapley, B. D., "Fundamentals of orbit determination," Theory of satellite geodesy and gravity field determination, Springer, 1989, pp. 235-260.

[4] Bisnath, S. B. and Langley, R. B., "Precise orbit determination of low earth orbiters with GPS point positioning," Proceedings of the 2001 National Technical Meeting of the Institute of Navigation, Long Beach, CA, 2001, pp. 725-733.

[5] Yunck, T., Bertiger, W., Wu, S., Bar-Sever, Y., Christensen, E., Haines, B., Lichten, S., Muellerschoen, R., Vigue, Y., and Willis, P., "First assessment of GPS-based reduced dynamic orbit determination on TOPEX/Poseidon," Geophysical Research Letters, Vol. 21, No. 7, 1994, pp. $541-544$.

[6] Kaplan, E. and Hegarty, C., Understanding GPS: principles and applications, Artech house, 2005.

[7] Morris, J., Zemerick, S., Grubb, M., Lucas, J., Jaridi, M., Gross, J. N., Ohi, N., Christian, J. A., Vassiliadis, D., Kadiyala, A., et al., "Simulation-to-flight (stf-1): A mission to enable cubesat software-based validation and verification," 2016.

[8] Montenbruck, O., Van Helleputte, T., Kroes, R., and Gill, E., "Reduced dynamic orbit determination using GPS code and carrier measurements," Aerospace Science and Technology, Vol. 9, No. 3,2005 , pp. 261-271.

[9] Chung, L. R., Orbit determination methods for deep space drag-free controlled laser interferometry missions, Ph.D. thesis, 2006. 
[10] Rim, H. and Schutz, B., "Precision orbit determination (POD)," Algorithm Theoretical Basis Document, Center for Space Research, The University of Texas at Austin, Austin, Tex, USA, 2002.

[11] Hobbs, D. and Bohn, P., "Precise orbit determination for low earth orbit satellites," Annals of the Marie Curie Fellowships, Vol. 4, 2006, pp. 128-135.

[12] Yunck, T. P., Wu, S.-C., Wu, J.-T., and Thornton, C. L., "Precise tracking of remote sensing satellites with the Global Positioning System," IEEE Transactions on Geoscience and Remote Sensing, Vol. 28, No. 1, 1990, pp. 108-116.

[13] Mehrparvar, A., Pignatelli, D., Carnahan, J., Munakat, R., Lan, W., Toorian, A., Hutputanasin, A., and Lee, S., "Cubesat design specification rev. 13," The CubeSat Program, Cal Poly San Luis Obispo, US, Vol. 1, 2014, pp. 2.

[14] Heidt, H., Puig-Suari, J., Moore, A., Nakasuka, S., and Twiggs, R., "CubeSat: A new generation of picosatellite for education and industry low-cost space experimentation," 2000.

[15] "Launch Vehicles," http://www.interorbital.com/Launch\%20Vehicles, (Accessed on $04 / 09 / 2018)$

[16] "Rocket Lab - Dedicated Satellite Launch - Rocket Lab," https : //www.rocketlabusa.com/ launch/dedicated/, (Accessed on 04/09/2018).

[17] "Deploying Small Satellites From ISS — NASA," https://www.nasa.gov/mission_pages/ station/research/benefits/cubesat, (Accessed on 04/09/2018).

[18] "J-SSOD:Experiment - International Space Station - JAXA," http://iss.jaxa.jp/en/ kiboexp/jssod/, (Accessed on 04/09/2018).

[19] Foster, C., Hallam, H., and Mason, J., "Orbit determination and differential-drag control of planet labs cubesat constellations," arXiv preprint arXiv:1509.03270, 2015.

[20] Cutler, J., Seitzer, P., Lee, C. H., Washabaugh, P., Sharma, S., Gitten, R., Piergentili, F., Santoni, F., Cardona, T., Cialone, G., et al., "Improved Orbit Determination of LEO CubeSats: Project LEDsat," Advanced Maui Optical and Space Surveillance (AMOS) Technologies Conference, 2017.

[21] Grzesik, B., Bestmann, U., and Stoll, E., "Beocube-A Platform for Flexible Precise Orbit Determination," 4S Symposium, 2016. 
[22] Kahr, E., Montenbruck, O., OKeefe, K., Skone, S., Urbanek, J., Bradbury, L., and Fenton, P., "GPS tracking on a nanosatellite the CanX-2 flight experience," Presentation to 8th presented at international ESA conference on guidance, navigation $\&$ control systems, Karlovy Vary, Czech Republic, June, 2011, pp. 5-10.

[23] Arlas, J. and Spangelo, S., "GPS results for the radio aurora explorer II cubesat mission," 51st AIAA Aerospace Sciences Meeting including the New Horizons Forum and Aerospace Exposition, 2013, p. 123.

[24] Yang, Y., Yue, X., Tang, G., Cui, H., and Song, B., "Orbit determination using combined GPS+ Beidou observations for low earth cubesats: Software validation in ground testbed," China Satellite Navigation Conference (CSNC) 2015 Proceedings: Volume III, Springer, 2015, pp. $321-334$.

[25] Schutz, B., Tapley, B., and Born, G. H., Statistical orbit determination, Academic Press, 2004.

[26] Misra, P. and Enge, P., Global positioning system: signals, measurements, and performance, Ganga-Jamuna Press, 2012.

[27] Thrun, S., Burgard, W., and Fox, D., Probabilistic robotics, MIT press, 2005.

[28] Blewitt, G., "An automatic editing algorithm for GPS data," Geophysical research letters, Vol. 17, No. 3, 1990, pp. 199-202.

[29] "Space-Track.Org," https://www.space-track.org/.

[30] Kulu, E., "The Nanosatallite Database," Nanosats. eu, 2016.

[31] GPSoft, "Satellite Navigation TOOLBOX 3.0 User's Guide," 2003.

[32] "GIPSY-OASIS," https://gipsy-oasis.jpl.nasa.gov/. 